\title{
REFLEXIONES SOBRE LA EDUCACIÓN INTEGRAL A PARTIR DEL PENSAMIENTO DE SANTO TOMÁS DE AQUINO
}

\author{
Faustino Corchuelo Alfaro, O.P. \\ Doctor en Teología Moral de la Universidad de Fribourg, Suiza \\ Rector de la Universidad Santo Tomás, Bucaramanga \\ faustinocorchuelo1@gmail.com
}

\begin{abstract}
Resumen
El presente artículo ofrece una reexión sobre la noble y difícil labor de educar, teniendo como fuente de inspiración el pensamiento losóco de Tomás de Aquino. ¿En qué consiste precisamente educar y, sobre todo, para qué se educa? ¿Qué se pretende educar en el ser humano y cuáles son los agentes responsables de este proceso? Hoy se entiende y se asume que dicha responsabilidad recae fundamentalmente en el propio educando, pero que en sus etapas iniciales y en las más aptas para incidir positivamente, como son la adolescencia y la juventud, juegan un papel preponderante el núcleo familiar y las instituciones educativas con sus profesores o maestros, a quienes corresponde la educatividad, es decir, la capacidad de ejercer inuencias positivas en el educando, de suerte que su aporte sea valioso en el desarrollo integral de ese ser humano en su fase de crecimiento.
\end{abstract}

Palabras clave: Educar, pensamiento losóco, desarrollo integral, educando, familia

\section{Summary}

This article is a reection on the noble and hard action of teaching, having Saint Thomas Aquinas philosophical thinking as inspiring source. What is teaching about and, above all, what do we teach for? What is it intended to educate in the human being and who is the responsible for this process? This is a responsibility that depends not only on the student himself but also on the family, especially in early stages, and the institutions with their professors and teachers who are the ones in charged of helping in this process through the educability or the capacity to inuence the student positively aiming at a unique contribution to the integral human being development in its growing phase.

Key words: Educate, Philosophical Thinking, Integral Development, Student, Family

En el pensamiento losóco-teológico de Santo Tomás aparece una serie de ideas inte- resantes que facilitan la comprensión del signicado del proceso educativo. No es que se encuentre en sus obras un tratado sistemático dedicado a la educación, se trata de reexiones densas dispersas en diversos tratados de su producción intelectual. Por ejemplo, encontramos textos que abordan la educación intelectual, tanto para mostrar el papel o causalidad del maestro ("De veritate" q. 11, más conocido como "De Magistro"), como para mostrar el papel o causalidad del discípulo (Santo Tomás, 1958, I, q. 117, a. 1 y 2; 1953, II, c. 75). Así mismo en las cuestiones que dedica en la Suma Teológica a la virtud de la estudiosidad y al vicio de la curiosidad (Santo Tomás, 1958, II-II q. 166 y 167). Hay innidad de textos referidos a la edu-

al papel de los padres en el cuidado de la prole (Santo Tomás, 1953, III, c. 122127 y paralelos). Su carta a un joven estudiante y su discurso o lectio inaugural como nuevo magister in Sacra Pagina en la Universidad de Paris, contienen ideas y pensamientos brillantes acerca de la educación .

\section{¿QUÉ ES ESO DE EDUCAR?}

La palabra educar posee una gama de signicados, algunos de los cuales son imprecisos, vagos o reduccionistas. En el lenguaje coloquial solemos decir o escuchar, por ejemplo, que una persona es muy educada, dando a entender con ello que dicha persona es de buenos modales

Las

palabras también tienen su historia, por lo que resulta interesante hacer una excursión etimo-

Existen dos autores clave de lengua hispana que han hecho una investigación seria y a fondo sobre las ideas fundamentales de Santo Tomás acerca de la educación: Antonio Millán Puelles y Enrique Martínez. 


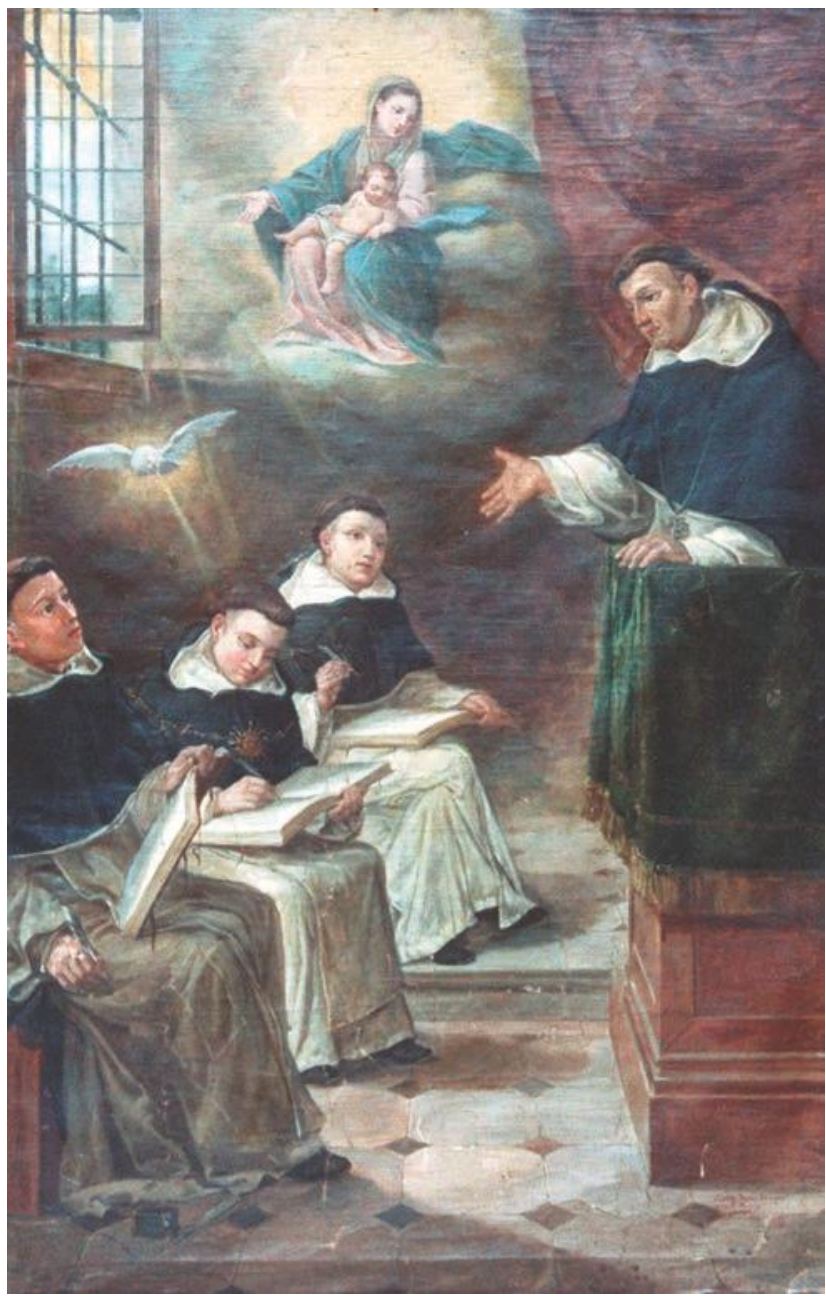

lógica por el término educar para ir descubriendo su primigenio signicado, pues ya desde los tiempos de Aristóteles se apuntaba como un principio básico para lograr la comprensión de la naturaleza de una cosa o de una acción, que el primer paso es descubrir el origen etimológico y el sentido que tienen las palabras con las cuales pretendemos signicar algo.

El término educar, etimológicamente, se deriva del latín "duco"="conducir", en cuya raíz está la palabra "dux" (jefe, general). Educar sería, entonces, conducir a alguien hacia un determinado objetivo, como un general conduce una guerra hacia la victoria. Al verbo "duco" lo precede la partícula "ex"="desde", signicando el compuesto de los dos "extraer", "conducir hacia fuera y hacia arriba, elevando" (Ernout \& Meillet, 1979, p. 186, 192, 204). Educar traduce, pues, la acción de sacar "hacia fuera", "sacar a la luz" lo que ya existe de alguna manera dentro del niño o del educando. Se trata de un proceso dinámico que debe conducir a extraer del adentro del individuo todo ese cúmulo de virtualidades y posibilidades que el ser humano posee en estado germinal y que debe sacar a ote para su proceso de realización personal, elevándose así a la plenitud de su ser. En otras palabras, educar es convertir a alguien en persona, dueña de sí misma y de su propio destino.

Generalmente, una denición consiste en una delimitación conceptual de lo que una cosa o acción es, puesto que denir es poner límites (del latín "de-nio"). Podríamos asumir como denición de educación, la que da Tomás en el Comentario al Libro IV de las Sentencias, en un contexto donde se pregunta si el matrimonio es algo natural o no. Allí el Aquinate entiende la educación como "la conducción progresiva y promoción hasta el estado perfecto de hombre en cuanto hombre"; esto signica que el educando debe ser guiado por etapas y en sentido ascendente hasta alcanzar el nivel de excelencia ("perfección"). Hablando de los tres nes del matrimonio, dice que uno de ellos se reere no simplemente a la procreación sino también a la educación de la prole: "La naturaleza no tiende solamente a la generación de la prole, sino también a su conducción y promoción (traductio et promotio) ${ }^{2}$ al estado perfecto del hombre en cuanto hombre, esto es, al estado de virtud" (Santo Tomás, 1996, In IV Sent. D. 26, q. 1, a. 1 in c). Nos encontramos, pues, ante un pensamiento denso, de una riqueza conceptual increíble.

\section{Educar: ¿Para qué?}

Santo Tomás, maestro de sabiduría, ha legado el arte de hacer preguntas pertinentes. Además de la pregunta inicial: ¿En qué consiste la tarea de educar? Surgen otras dos preguntas adicionales, ¿Para qué se educa? Y: ¿Quiénes

2 El término "traductio" tiene una clara cercanía etimológica con "educatio", pues coinciden en la raíz (-duco), diferenciándose en el prejo (trans - ex). Traductio signica un mero conducir más allá, de un sitio a otro, sin la connotación de elevación que aparece en educatio y que también implica la promotio. 
son los agentes responsables de este proceso? Así pues, una pregunta clave que debería siempre plantearse es: ¿Educar, para qué? Es necesario partir de la pregunta esencial en torno al " $n$ " ("telos" en griego) que se persigue con la educación; sólo una vez que se responda a esta pregunta será posible resolver los problemas derivados de los medios. Tomás recurre al método de análisis de las cuatro causas (material, formal, eciente y nal), pues ellas expresan de manera más o menos precisa aquello que se pretende comprender. Sin embargo, privilegia en el caso de la educación, la causa nal, puesto que ella es la primera en un orden de prioridades.

Antes que pensar, pues, en cuestiones acerca de los medios y agentes que intervienen en el proceso educativo, práctica común en el ámbito educativo, para él convendría tener claro el $n$ que se persigue con la educación. Lo que realmente interesa no es tanto saber cómo educar sino el para qué educar, tener el coraje de plantearse grandes preguntas referidas a los nes y no a los medios. Los libros, los medios audiovisuales, la estructura del sistema educativo, los talleres, los seminarios son meros instrumentos al servicio del crecimiento personal de cada estudiante. Haber olvidado esta regla fundamental, invirtiendo la relación entre medios y nes es lo que denuncia con vigor Paul Ricoeur cuando habla de la hipertroa de los medios y la atroa de los nes que caracteriza nuestra sociedad. Una sociedad que olvida los nes y se vuelca sobre los medios hipervalorándolos, corre el riesgo de perder su brújula y no saber hacia dónde se dirige (Poupard, 2005).

La pregunta clave sobre el para qué nos induce a analizar distintos aspectos y a meditar acerca del sentido de la actividad educativa. Al hacer este ejercicio podríamos encontrar dos tipos de educación: la que enseña a ga-

3 Causa material de la educación: Como el verbo enseñar exige doble acusativo, la primera materia es lo que se enseña, y la otra es aquel a quien se enseña; causa formal: la acción misma de conducir y promover al estado de virtud; la causa eciente: el mismo educando, sus padres y maestros; causa nal: la consecución del estado perfecto en cuanto hombre, que es la virtud. narse la vida y la que enseña a vivir. La primera se da cuando se reduce la educación a la mera instrucción, cuando se educa sólo el intelecto con la mira puesta en la adquisición de conocimientos, la competitividad, la productividad y el rendimiento económico con un enfoque exclusivamente profesionalizante; la segunda tiene lugar si, por el contrario, inculcan valores para que el joven asuma con responsabilidad su proyecto de vida, para rearmar su voluntad de seguir aprendiendo durante toda la vida, para saber afrontar la complejidad, las exigencias y las incertidumbres de la vida, para incidir positivamente en el desarrollo humano armónico, justo y solidario de la comunidad, de su entorno.

El propósito de todo proceso educativo no es sólo crear seres humanos libres, lúcidos, armoniosos y expresivos, sino seres con un sentido profundo de pertenencia a una comunidad. La competitividad extrema estimula el egoísmo, los ejercicios de cooperación estimulan nuestra conciencia de que necesitamos de los otros, fortalecen nuestro sentido de comunidad (Ospina, 2012, p. 49).

En otras palabras, la educación nos permite crecer como seres humanos, incorporarnos de la mejor forma a la sociedad y aportarle a ella lo mejor de uno mismo.

Así, pues, el gran objetivo que debe perseguir la educación debe ser, ante todo, la conducción y la promoción del hombre a un estado perfecto que, según Santo Tomás, es el de la virtud. Es decir, la educación debe ayudarle al niño, la niña o al joven para que asuma la construcción de su propio destino, de formarse a sí mismo como ser humano. Formar-se es la forma pronominal reexiva de "formar", que corrige y purica la idea de pasividad, de una imposición externa, para asumir por propia cuenta la aventura de darse su propia forma u horma, de descubrir el tesoro de su realización personal. Formarse signica, pues, ascender en humanidad, llegar a ser en plenitud, 
dar forma a las disposiciones y capacidades naturales que subyacen en estado germinal (Libanio, 2003).

¿Qué querría decir Santo Tomás cuando armaba que la educación consiste en conducir y promover al hombre a su estado perfecto en cuanto hombre? Estado perfecto es sinónimo de algo totalmente hecho, acabado, estable, sin tacha, cualidad que solamente es aplicable a Dios, pues el ser humano es siempre perfectible, en proceso de mejoramiento continuo, o también defectible, en proceso de degradación o desmejoramiento. Llegar a ese estado perfecto en cuanto hombre, que es el estado de virtud, no es algo que se logra de la noche a la mañana, sino que se trata de una empresa que abarca y dura toda la vida, de un proceso de lenta maduración, de laboriosa y constante disciplina, que no se agota con una meta u objetivo conseguido.

El espíritu humano es "devenir", vocación de "llegar a ser". Como no hay coincidencia entre el "ser" y el "deber ser", se puede armar que el hombre no es por naturaleza lo que debe ser y, justamente por ello, necesita de la formación, de la educación. Además, el hombre, por su carácter de espíritu encarnado, está en cierto modo inacabado, imperfecto aunque perfectible. Siempre está y estará insatisfecho con lo que es, con lo que sabe y con lo que tiene, y andará a la búsqueda de algo nuevo, de algo más perfecto, más plenican te. La satisfacción de cualquier necesidad, lejos de apaciguar, crea una tensión hacia otra cosa; cada necesidad saciada hace brotar otra. Por eso, su vida requiere un nuevo tipo de perfección que no sea ya substancial sino accidental, puesto que no se trata ni basta con el simple "ser" ("esse") sino que lo ideal es alcanzar "el ser bien" ("bene esse"), por ejemplo, ser honesto, honrado, justo, solidario, puntual o sabio.

Así, la educación debe procurar habilitar y perfeccionar todas las potencias o facultades del ser humano que lo disponen para lograr el estado perfecto o plenitud humana, su "bene esse" y que Santo Tomás llama el estado de virtud. La virtud (dynamis en griego) la dene el Doctor Angélico como un hábito operativo del bien, es decir, una disposición estable que ayuda a obrar pronto, fácil y placenteramente (prompte, faciliter, delectabiliter) lo debido. Pero es un hábito que hay que adquirir.

Por ejemplo, una persona virtuosa en el arte de tocar el violín o cualquier otro instrumento musical, en sus inicios debió esforzarse para lograr algunas notas quizá con torpeza, pero con el tiempo y un trabajo constante (disciplina) adquirió la virtud de ejecutar 0 componer una pieza musical con facilidad y con gusto, de tal suerte que se convirtió para ella en algo connatural y gratican te. De la misma manera, mediante un trabajo disciplinado y constante, un ser humano puede adquirir cualquier virtud tanto en el orden intelectual como en el moral. La plenitud de lo humano está en la adquisición de los hábitos buenos o virtudes. A la persona que es capaz de hacer sonar una auta, el arte la llevará a hacerla sonar con maestría; a la persona capaz de practicar la justicia o cualquier otra virtud, esta la llevará a hacerlo de un modo connatural, con facilidad e incluso con placer. Así mismo, a la persona que cultiva los hábitos intelectuales, esto la conducirá a la adquisición de la ciencia y la sabiduría sin mayores icultades y con cierta fruición espiritual, el gozo de buscar y encontrar la verdad, es decir, gaudium de veritate.

Puesto que la virtud perfecciona la naturaleza, con razón se le ha llamado una segunda naturaleza. En efecto, por la forma sustancial (el alma) la materia corpórea llega al "esse" (ser), es decir, a "ser hombre", y por la práctica de la virtud se llega al "bene esse", ("ser hombre de bien) vale decir, se logra un valor agregado al ser simplemente una mujer o un hombre: sabio, bueno, honrado, honesto, justo, prudente, magnánimo, solidario. 
Desde esta óptica, al interrogante educar ¿para qué? se podría responder: para lograr mejores seres humanos y una mejor sociedad. En otras palabras, la educación nos permite crecer como seres humanos, incorporarnos en forma positiva a la sociedad y darle a ella lo mejor de nosotros mismos, tener una losofía de la vida que incluye creencias, valores, visión, propósitos, motivaciones y actitudes que le dan sentido y valor a la propia existencia.

Para formar mejores seres humanos se adopta, entonces, el enfoque de una formación integral, cuya idea maestra consiste en desarrollar de manera armónica y equilibrada todas las potencialidades físicas, artísticas, intelectuales, morales y espirituales inscritas en la naturaleza humana y que conguran la personalidad de los individuos. Escribe el siquiatra español Enrique Rojas:

Educar es ayudar a alguien para que se desarrolle de la mejor manera posible en los diversos aspectos que tiene la naturaleza humana(...). Educar signica comunicar conocimiento, despertar habilidades y promover actitudes(...). Información y formación constituyen un binomio clave en toda educación. La primera abre la puerta, y la segunda nos instala en el proceso educativo. Son dos etapas sucesivas y complementarias. No hay educación completa si falta alguna de ellas. Recibir información es acumular una serie de datos, observaciones y manifestaciones especícas. La formación va más allá: ofrece unos criterios para regir el comportamiento, de acuerdo con una cierta orientación; pretende sacar el mejor partido posible

de los conocimientos recibidos, favoreciendo la construcción de un hombre más maduro, más sólido y rme, más humano y más espiritual, más dueño de sí mismo (Rojas, 1999).

A través de la educación se desarrolla un proceso de mejoramiento personal contínuo de todas las dimensiones de la vida humana (biológica, física, emocional, afectiva, intelectual, espiritual, laboral, profesional, familiar, social y cultural, entre otras) de suerte que permita comprender mejor el mundo, saber convivir e interrelacionarnos con los demás, trascender y avanzar hacia nuestra plena realización.

\section{Educar ¿Qué?}

En principio, al ser humano en su totalidad: la corporeidad, la capacidad motriz, la sensibilidad, los sentimientos, pero fundamentalmente las dos facultades eminentemente humanas que nos distancian de los seres vivos no humanos, como son el pensamiento reexivo o capacidad de razonar y la voluntad libre. Los seres vivos no humanos tienen una existencia cuyo sentido está predeterminado, pues les viene impuesto por la naturaleza o por el instinto, vale decir, su vida tiene para cada uno de ellos un sentido monovalente, bien programado 
desde el comienzo de su existencia. En cambio, el ser humano es el único ser vivo en el mundo que tiene la capacidad de reexión, es decir, la capacidad de interrogarse y de pensar sobre sí mismo, sobre su existencia y darle así una orientación y un sentido a su vida. Dotado de pensamiento reflexivo y de una voluntad libre que le permite abrirse a una extraordinaria multiplicidad de sentidos y de orientaciones diversas, ser dueño de labrar su propio destino.

Dar un sentido a la vida signica asumir la propia vida, poner orden y tomar las opciones fundamentales que polaricen y marquen el derrotero de su existencia. "El hombre no es comparable a un árbol o a un animal que no conocen ni porvenir ni vocación(...). Es un ser que se interroga. Ha de tomar su vida en sus manos y buscarle sentido" (Schillebeeckx, 1965). La libertad humana nace de la conuencia del pensamiento reexivo que conoce y juzga y la voluntad que desea, quiere y ama espontáneamente el bien. Claro que el privilegio de la libertad implica el riesgo de ejercerla erróneamente, al asumir opciones o buscar nes que pueden ser destructores o degradantes, tanto para el individuo como para la comunidad.

Se abre pues, ante nuestros ojos, todo un vasto horizonte de virtudes propias del ser racional que pueden y deben ser objetos de educación, pero que fundamentalmente podemos agrupar en dos: las virtudes intelectuales, que perfeccionan el entendimiento en su capacidad de conocer y descubrir la verdad, y la virtudes morales, que perfeccionan la voluntad y habilitan a la persona para ser un buena y honesta. Trabajos recientes desarrollados desde la psicología muestran la relevancia de la enseñanza de las virtudes en la sociedad contemporánea (Gardner, 2012).

Las virtudes intelectuales ("dianoéticas" en griego) que pueden adquirirse, ya sea por vía de infusión o de la invención y por la vía de la enseñanza-aprendizaje, son de dos órdenes, a saber, especulativas, esto es, ordenadas al conocimiento de la verdad; y de carácter prácti$c o$, esto es, ordenadas a la realización de una acción o al obrar propiamente humano. Las virtudes intelectuales especulativas prácticamente son dos: la ciencia y la sabiduría.

La ciencia, es decir el conocimiento propiamente cientíco que perfecciona el raciocinio, que discurre a partir de principios universales o particulares en tal o cual campo de los seres cognoscibles, tales como las ciencias exactas, económicas, sociales y humanas, entre otras, y de las cuales se pretende desentrañar la verdad que contienen con miras a una nalidad puramente teórica u ordenadas a una nalidad de carácter práctico.

La sabiduría, a partir de los principios universales últimos y con una mirada abarcadora, juzga y ordena la vida y cualquier conocimiento cientíco, tanto en sus principios como en sus conclusiones, de suerte que puede armarse que constituye el principal hábito intelectivo. "El estudio de la sabiduría -arma Santo Tomás- es el más perfecto, sublime, provechoso y alegre de todos los estudios humanos" (1996, I, c. 2, n. 1). Sin esa visión globalizante que proporciona la sabiduría, la educación puramente cientíca del intelecto no sólo permanecerá incompleta, sin norte ni brújula, con el grave peligro de perderse en el laberinto de las ciencias, las cuales a veces tienden a ponticar desde su punto de vista o a sustituir el vacío dejado por la enseñanza y la adquisición de la sabiduría, pretendiendo ser guía de la vida y de las demás ciencias, partiendo desde principios particulares o reduciendo su enfoque a lo mero práctico y, sobre todo, técnico (Martínez, 2002).

En el seno de la familia se puede adquirir, de alguna manera, cierta sabiduría espontánea que llevará al niño y al joven a descubrir un rico depósito de verdades fundamentales y de carácter trascendental que, en el curso de 
su misma existencia, le servirán de luz y de criterio para descifrar y juzgar rectamente la realidad. Verdades acerca del sentido de la propia vida, del sufrimiento, de la sexualidad, de la muerte, del misterio del mal y, sobre todo, de ese misterio insondable que es Dios, verdades que afectan todo lo que pasa en el amplio mundo de la historia o en la pequeña historia personal. Sabiduría espontánea que en la edad madura se debe reforzar y consolidar con argumentos más profundos y convincentes. En el plano religioso, la sabiduría aparece como el don supremo ligado a la caridad (Santo Tomás, 1996, II-II, q.45).

Según Santo Tomás, junto a las virtudes especulativas encontramos otras dos virtudes intelectuales ordenadas ahora a lo práctico, como son el arte y la prudencia. Se diferencian entre sí, en que la primera será aquella disposición innata o adquirida ordenada más a perfeccionar las habilidades fácticas o estéticas del ser humano; mientras que la segunda se trata de un saber orientado a cómo obrar responsablemente en una situación concreta. Es esa especie de "sabiduría acerca de las cosas humanas" que invita a prever el futuro y a tomar en serio el presente.

Pero las virtudes que perfeccionan al entendimiento humano en orden a conocer mejor la verdad de las cosas, no son las únicas que son perfectibles y educables, sino que existen aquellas que perfeccionan la voluntad, entendida ésta como la facultad que inclina espontáneamente al hombre a la búsqueda y realización del bien universal, por lo que los escolásticos la denominaban "el apetito espiritual cuyo objeto es el bien universal conocido por la razón”. Esa inclinación espontánea de la voluntad hacia el bien universal se encuentra en estado de indeterminación, es decir, que requiere el cultivo de virtudes o hábitos operativos que la determinen convenientemente a querer, buscar y realizar ese bien universal. La voluntad se educa entrenándola día a día, estableciendo una verdadera jerarquía de valores y de proyectos,

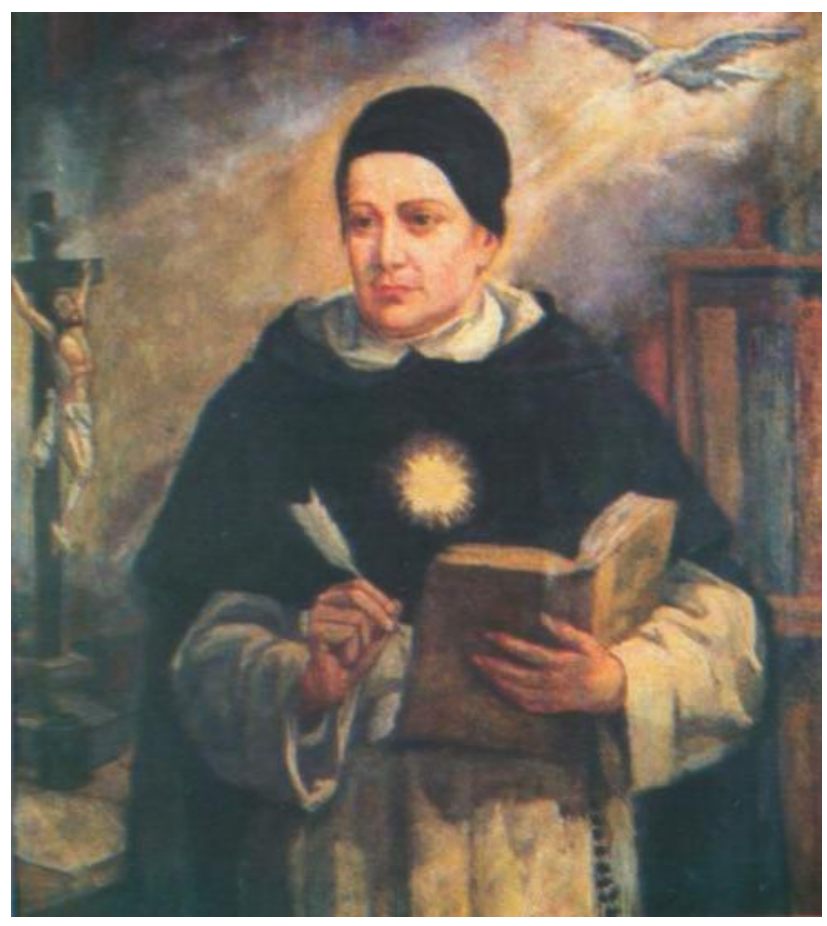

poniendo orden en el campo de los deseos y en el laberinto de las pasiones.

Las principales virtudes que perfeccionan y habilitan la voluntad para que la persona sea buena y transforme todo lo que hace en una operación buena, son las virtudes morales. La clasificación de las virtudes morales, que realmente son muchas, ha sido reducida en la tradición filosófica, las ha restringido a las llamadas virtudes cardinales, porque en torno a ellas giran todas las demás como si fueran sus quicios. Entre estas se encuentran aquellas relacionadas con la sensibilidad y el mundo pasional, y esas virtudes son la templanza y la fortaleza, que van a poner orden y moderar las tendencias innatas a la conservación de la propia vida como de la especie, la inclinación hacia lo fácil y placentero, lo mismo que a templar el espíritu para las dificultades de la vida. La persona no es simplemente un ser espiritual o angelical, sino un animal y descuidar la educación en el ámbito de las pasiones implica dejar a la voluntad desamparada a la hora de realizar sus operaciones, pues las pasiones desordenadas pueden ennubilar la mente e impedir el recto ejercicio de una acción buena. Así, 
la educación consistirá en hacer del ser humano un animal ético. La educación debe ser, sobre todo, la educación de los deseos y de la afectividad (Rojas, 2004). Un adecuado cultivo y una adecuada práctica de estas dos virtudes cardinales, conseguirá una bene ciosa armonía entre la voluntad y la sensibilidad que corresponden a su condición animal.

Como el ser humano es un ser de relaciones, existe otra virtud cardinal clave que va a regular la red de relaciones íntimas y profundas que el ser humano teje con los demás congéneres para la construcción de su propio proyecto de realización personal. Se trata de la justicia la cual tiene como objeto regular las operaciones de la voluntad en orden a dar a cada uno lo que le es debido y que permite a la persona ser constructora de un orden justo y armónico en las relaciones humanas.

Por último, existe una virtud moral mucho más cerca de la razón que la templanza, la fortaleza y aún que la misma justicia. Es la virtud de la prudencia. Ella nos permite deliberar correctamente, mostrándonos lo más conveniente en cada momento de nuestra vida. No lo más conveniente a corto plazo, sino lo más conveniente para una vida buena en su totalidad. La prudencia nos facilita el discernimiento en la toma de decisiones, guiándonos hacia el logro de un equilibrio entre el exceso y el defecto y como especie de guía de las demás virtudes. Siendo una virtud intelectiva que busca ayudar al entendimiento a razonar de un modo adecuado y constructivo, también es una virtud moral, la primera de las virtudes cardinales, aquella debe tomar las riendas de las demás virtudes humanas ("Auriga virtutum", la llamaban los lósofos romanos), de suerte que favorezca la plena realización de la persona. La grandeza del ser humano consiste en ser dueño de sí mismo (autónomo), que pueda prever y proyectar su propio destino (“ipse est sibi providens”), asumir lo que quiere llegar a ser, tener la capacidad de decidir e inventar acciones que transformen la realidad y a él mismo. El dinamismo de la virtud de la prudencia le servirá de gran ayuda para ver y prever a través de la incertidumbre de la vida cotidiana. De ella dice Santo Tomás, con una hermosa expresión, es "la sabiduría acerca de las cosas humanas” (1996, II-II, q. 47, a. 2, ad 1), una especie de genio para la vida práctica.

Como se puede deducir, el cultivo de estas cuatro virtudes goznes, que llevan implícitas otras tantas, constituye la trama de la educación moral integral, tan fundamental para la sana y positiva convivencia en la sociedad humana, pero tan descuidada o relegada a un segundo plano en el proceso educativo en la infancia y la juventud. La pedagogía de la antigüedad se preocupaba más por la formación humana, por el cultivo de las virtudes morales y las cualidades y habilidades de cada uno. Las escuelas modernas han invertido el orden y han relegado a segundo plano la educación del ser cívico y moral para interesarse más en el cientíco, artesano o artista.

La promoción humana integral consiste en lograr que el educando adquiera las virtudes intelectuales, morales, técnicas o artísticas, que hacen al sabio, al cientíco, al virtuoso, al genio. No obstante, la virtud moral es la que hace realmente buena a la persona. Es ella la virtud por antonomasia. Sin esta no hay plenitud en lo humano. El excesivo culto al valor de la racionalidad, derivado de la losofía cartesiana y un enfoque unidimensional de la educación ha llevado a privilegiar en el sistema educativo la formación del intelecto mediante la instrucción, descuidando o dando escasa relevancia a la formación moral o al cultivo de las virtudes que perfeccionan la facultad de la voluntad en orden que el ser humano sea un animal ético. Incluso ya en tiempos de Sócrates se pensaba que para que éste fuera bueno y tuviera un comportamiento ético aceptable bastaba con sacarlo de la ignorancia instruyéndolo. Mas la experiencia ha comprobado que es insucien te la mera instrucción o educación del intelecto; hoy día diríamos que la 
mera capacitación profesional, sin cultivar las otras facultades y dimensiones del ser humano, no basta para sacar adelante los proyectos de la realización personal de cada individuo y por ende, la comunidad humana.

\section{AGENTES RESPONSABLES DEL PROCESO EDUCATIVO}

La responsabilidad es la capacidad de responder por sí mismo y de sí mismo. El verbo responder y todos sus derivados presentes en las lenguas romances proceden del verbo latino "spondeo", cuyo primer sentido es el de asumir un compromiso solemne. El más frecuente era aquel en el que el padre se compromete (spondet) a entregar a su hija (sponsa) en matrimonio. En esto consistía la ceremonia de los "esponsales" (sponsalia). De "spondeo" deriva "respondeo" que signica responder en el sentido preciso de cumplir un compromiso solemnemente asumido. La responsabilidad es, pues, la cualidad o condición de quien promete 0 se compromete. Existen dos tipos de responsabilidad: una personal e intransferible y otra compartida o de grupo, llamada también corresponsabilidad. Las dos pueden ser "fuertes" o morales y "débiles" o jurídicas. En la ética de la responsabilidad existen distintos tipos y grados de responsabilidad. En primer lugar, existe la responsabilidad personal, que es completamente intransferible y la responsabilidad compartida, o también llamada corresponsabilidad, la cual se da en grados diversos y en una doble dirección, horizontal y vertical, es decir, de una responsabilidad compartida tanto con los coetáneos y con quienes se comparte la vida como con el futuro de la sociedad y de las nuevas generaciones 4 .

\section{$1^{\circ}$ El propio educando}

¿Sobre quién recae, entonces, principalmente el peso de la responsabilidad del proceso educativo? Como se trata de un proceso que

4 Para profundizar en la reexión sobre la responsabilidad, se puede ver el análisis que hace Sedano (2012) en su libro Hacia una pedagogía de la respuesta, especialmente en los capítulos I al IV. normalmente dura toda la vida y es de lenta maduración, el mismo individuo, a medida que va aorando la conciencia reexiva y en forma progresiva, surge como el principal agente responsable de dicho proceso, (Santo Tomás, 1996, I, q. 117, a. 1 y 2; 1953 II, c. 75), de tal suerte que no puede endosar en la conciencia de los demás agentes todo el peso de la responsabilidad, sobre todo cuando termina afectando negativamente su proyecto de vida. La responsabilidad es, ante todo, personal e intransferible.

\section{$2^{\circ}$ Los padres y el ambiente familiar}

No obstante, otros agentes, instancias y factores tienen incidencia en este proceso, sobre todo en las etapas iniciales. Entre todos los agentes responsables del hecho educativo, Santo Tomás asigna un papel primordial a los padres y el ambiente hogareño, que es el lugar propicio y privilegiado para la educación del ser humano. Santo Tomás lo expresa bellamente cuando arma que "el hijo una vez que ha salido del útero materno estará bajo el cuidado de sus padres, como contenido en un útero espiritual" (Santo Tomás, 1996, I-II, q. 58, a. 2). En efecto, son quienes engendran al hijo, dándole el "ser" ("esse") y todo su patrimonio genético, son los principales responsables de proporcionarle alimento ("nutrimentum") y una crianza adecuada, es decir, ayudarlo a adquirir las actitudes fundamentales (valores) para "ser hombre de bien" ("bene esse"), y que más tarde necesitará para caminar por el sendero de la vida y hacerle frente a las vicisitudes de la misma.

El Doctor Angélico sitúa la educación como una prolongación de la generación. Para Aristóteles, explica Tomás, el bien de la prole no se logra con la obtención del ser, con el mero hecho de engendrar, sino que se requiere el proveer alimento -"nutrimentum"- y una buena crianza-"educatio" de los hijos, hasta que estos logren alcanzar la edad adulta, es decir, hasta que se conviertan en personas capaces de valerse por sí mismas con plena autonomía. 
Entre todos los animales, el racional es el que nace y dura un más tiempo en estado de precariedad e indigencia total. El ser humano, para lograr la madurez y plenitud de su existencia, requiere desde las primeras etapas de la vida del concurso indispensable tanto del padre como de la madre. Si falta uno de los dos o no concurren debidamente en el cumplimiento de este deber, la tarea y el objetivo quedarán truncados, o bien se cumplirán a medias. Hay especies animales -explica Santo Tomás- en las que la hembra se basta para alimentar a las crías y otras en las que no, como sucede en algunas especies de aves, que necesitan la presencia del macho. Es evidente que en la especie humana no basta la mujer sola para la crianza de los hijos, ya que las necesidades de la vida humana requieren tanto que no pueden ser satisfechas sólo por uno (Santo Tomás, 1996, III, c. 122, n.6). El estado de indigencia de la prole, en cuanto prole, debe ser satisfecha tanto por la crianza como por la educación. La primera sólo terminará cuando el niño ya se valga por sí mismo en el orden físico, y la segunda cuando ya se valga por sí mismo en el orden intelectual y moral.

El "estado de virtud" que propone el Aquinate como meta y $n$ de la educación, lo podemos calicar ahora, en consecuencia, como la llegada a la mayoría de edad. Esta mayoría de edad no se reduce a la mera madurez biológica sino que implica y abarca los demás componentes y dimensiones del ser humano: lo afectivo, lo intelectual, lo moral y lo espiritual, en los cuales debe crecer hasta alcanzar su plenitud. Se trata pues, de favorecer y promover el crecimiento integral de la persona humana.

En todo proceso formativo hay dos factores, la enseñanza y el aprendizaje. Educar consiste en enseñar algo a alguien, que a su vez lo aprende. En todo ser humano se dan tres procesos de aprendizaje distintos, con vías, ritmos y cronología muy diversos entre sí. No se aprenden del mismo modo los conocimientos, las habilidades y las actitudes. Estas tienen la característica de aprenderse muy temprana- mente; algunas, quizá las más importantes, en la primera infancia. En la educación inicial se desarrollan las capacidades psicofísicas de los seres humanos, es la etapa en que el cerebro y el sistema neuronal se están acabando de formar y en donde las actitudes éticas primarias, como ser honesto y honrado, decir la verdad, respetar al otro y ser solidario, se empiezan a arraigar. En esta etapa los padres y el ambiente familiar juegan un papel vital y son la base de toda la educación posterior.

Inspiradas en los griegos, quienes consideraban que la vida estaba dividida en escalones ("Clímax" = grada o escalera por la que se sube), la antropología y la psicología evolutiva del hombre medieval también clasicaba el crecimiento psíquico-biológico por etapas de septenios (Aristóteles, trad. en 1873). Cada siete o múltiplo de siete era una época crítica o climatérica, especialmente la de $7 \times 7=49$ y el $7 \times 9=63$, que son las edades climatéricas por excelencia. Las personas avanzan a través de distintos estadios a medida que van madurando biológica y humanamente. El primer estadio abarca desde el nacimiento hasta los siete años y se denomina infancia, es la etapa del desarrollo psicomotor, del desarrollo del lenguaje no verbal y verbal, del desarrollo intelectual, el desarrollo afectivo, cuyos dos primeros ingredientes son la risa y el llanto. En una palabra, es la etapa de la crianza. De los siete a los catorce va la segunda etapa y se denomina puericia o pubertad, en la que la condición corpórea permite al niño el uso de la razón más explícito y echar las bases para saciar su sed de conocer y de saber, eso sí dependiendo de la disciplina y enseñanza por medio de otro, que es el maestro. Es la etapa de la vida en donde la personalidad va adquiriendo ya un perl bastante denido. La tercera etapa o adolescencia se inicia a los catorce y en ella el adolescente va adquiriendo gradualmente una relativa autonomía que le permite asumir ciertos compromisos. La cuarta etapa, denominada juventud, arranca a partir de los veintiún años y supone el alejamiento denitivo 
de la niñez. Esta entrada en la edad adulta, por la que el hombre deja de estar bajo la tutela de los padres, supone una madurez que se traduce en la disminución de su educabilidad. Así, pues, la labor educativa de los padres va desde el nacimiento, pasando por la crianza en las primeras etapas de la vida hasta que los hijos se valgan por sí mismos en la edad adulta, y se caracteriza por la profundidad e intimidad necesarias para

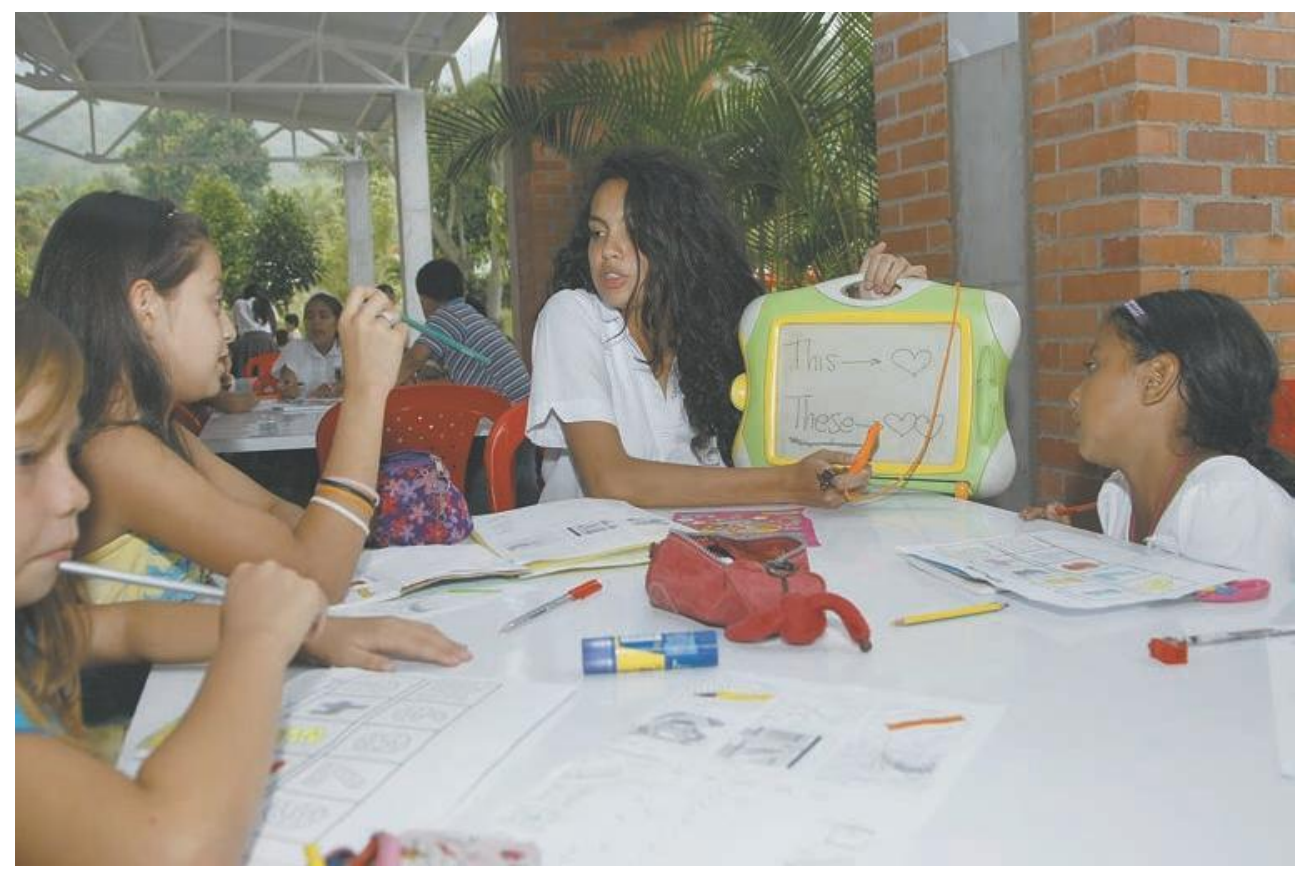
el crecimiento de sus hijos, condiciones favorables que no brindan la escuela o la vida social.

\section{$3^{\circ}$ Los maestros}

A este carácter primordial de responsabilidad educativa de los padres, sigue la de aquellos que suplen la indigencia e incapacidad de brindar una educación integral, tarea que recae en las instituciones educativas, en los maestros e indirectamente, en los dirigentes sociales y los medios de comunicación.

Después de la familia, la escuela y sus maestros complementarán la educación que el niño ha recibido en ella, tanto en su formación intelectual como en su formación cívica estética, moral y espiritual. La educación escolar es la que transmite la herencia cultural de la humanidad y la que debe cultivar ciertas habilidades y competencias que posteriormente le permitirán al niño y al adolescente comprender las ciencias y las normas para convivir con sus congéneres. Como se ha mencionado párrafos atrás, la educación no puede centrarse exclusivamente en la mera formación intelectual, pues el niño y el adolescente apenas están forjando su personalidad moral, intelectual y espiritual, por lo que necesitan de una consolidación, del reforzamiento de la formación en valores brindada por sus padres y en su ambiente familiar.

La etapa de la juventud y de la entrada a la edad adulta, que hoy en día coinciden con el ingreso y la permanencia en la universidad, no tiene la misma función pues se supone que el joven que ingresa en ella ya debe haber alcanzado una cierta autonomía y madurez moral, aunque en la realidad esto se da solo en un relativo grado. En esta etapa la educación y la capacidad simbólica le permitirá la joven conocer, entender, criticar, abstraer, innovar, relacionar y transformar la información recibida para comprender y transformar igualmente el mundo y la condición humana. Es por ello que la universidad se dedicará más a su capacitación profesional, al cultivo de las virtudes intelectuales mediante la enseñanza y la investigación. Ello no signica que haya que relegar al olvido la educación de las otras dimensiones fundamentales de la vida. La formación cientíca de la universidad tampoco deberá perder de vista el ideal último de saber especulativo, concretado en la virtud de la sabiduría. Si la familia es el lugar propio de la sabiduría espontánea, la universidad debe ser el del ambiente propicio de la sabiduría cientíca o saber sapiencial, en donde los logros cientícos o tecnológicos 
están acompañados de una visión ética y una cosmovisión losóca de los mismos.

Al educador, en cualquiera de sus niveles, le corresponde la educatividad, es decir, la capacidad para ejercer inuencias positivas en el educando para contribuir así al desarrollo integral del ser humano en su fase de crecimiento. Ejercer inuencias positivas en el educando conlleva la voluntad de guiarse por unos principios pedagógicos que iluminen la gestión educativa. En primer lugar, tener clara conciencia que el maestro en el proceso educativo no juega más que un papel subsidiario, ministerial en el pleno sentido de la palabra, es un Minister naturae o ministro de la naturaleza, como lo llama Santo Tomás. Es decir, se trata de alguien que ayuda o sirve desde fuera para que el educando se realice vitalmente desde dentro y logre así el despliegue de sus capacidades germinales. El maestro es como el médico. Éste ayuda a los procesos naturales del enfermo, evita lo nocivo y corrobora lo que favorece la salud. Lo mismo ha de hacer el maestro.

A propósito de esto, cuando hablamos de perles hacemos alusión a una especie de desideratum que apunta hacia lo óptimo. En el caso del perl del maestro, éste debería incluir, además de dominio de la ciencia o disciplina que enseña, excelentes relaciones humanas y un lenguaje actitudinal positivo. Esto es de gran trascendencia, pues con su lenguaje actitudinal, el docente debe poner de maniesto que busca, ante todo, el bien de sus alumnos para que ellos se sientan tratados como personas, es decir, con consideración y respeto, con un profundo sentido de justicia.

El verbo enseñar en latín exige un doble acusativo: la materia que se enseña y el sujeto a quien se enseña. Esto quiere decir que en el proceso educativo debe existir cierto interés tanto por el mensaje o contenido que desea transmitirse, como por la persona destinataria o el sujeto de la educación. La enseñanza no es sólo un proceso de transmisión de conocimien- tos sino también, y principalmente, de aprendizaje. Todo lo que incida en la vida de los estudiantes debería preocupar y hacer reexionar a los docentes: su competencia pedagógica, el dominio de la materia que enseña, la mística que tiene por su profesión, su cultura general, sus cualidades humanas y morales, su manera de evaluar, entre otros aspectos importantes, porque todo tiene una incidencia y repercusión decisiva en la vida de ese ser humano que está en proceso de formación.

El maestro logra promover la plenitud del discípulo de dos modos: con la doctrina propia de cada ciencia o disciplina, por la cual le indica dónde está la verdad, el bien, la belleza. La doctrina se debe poseer, en la medida de lo posible, en grado sumo para poderla transmitir de modo convincente. Esto supone algo que, como se ha dicho antes y aunque parezca evidente, no siempre se da: el maestro debe saber y tener un dominio sobre aquello que enseña. Debe tener autoridad, una autoridad epistemológica, propia de quien se ha apropiado un saber especíco, lo ha actualizado y enriquecido además, lo sabe transmitir con arte a quienes están ávidos de aprender. Quien se dedica a enseñar debe tener esa vocación de servicio, buscar que el estudiante saque el mayor provecho de aquello que se le enseña, de tal forma que propicie su crecimiento integral. De esta verdad se desprende, como un corolario, que el docente debe ser claro y didáctico utilizando adecuadamente los términos, preparando convenientemente el razonamiento, de tal forma que no genere confusión en sus alumnos, sino todo lo contrario, claridad. Como señala Chesterton (1996) "una idea que no es clara, no es buena idea; una palabra que no se puede comprender, no es una buena palabra".

Un maestro tiene que ser como una especie de tutor, de asesor, de orientador, un guía, un facilitador, un provocador de ideas y proyectos, una persona cuya palabra o presencia incite a una respuesta estimulante y productiva con aquellos con quienes interactúa, alguien que 


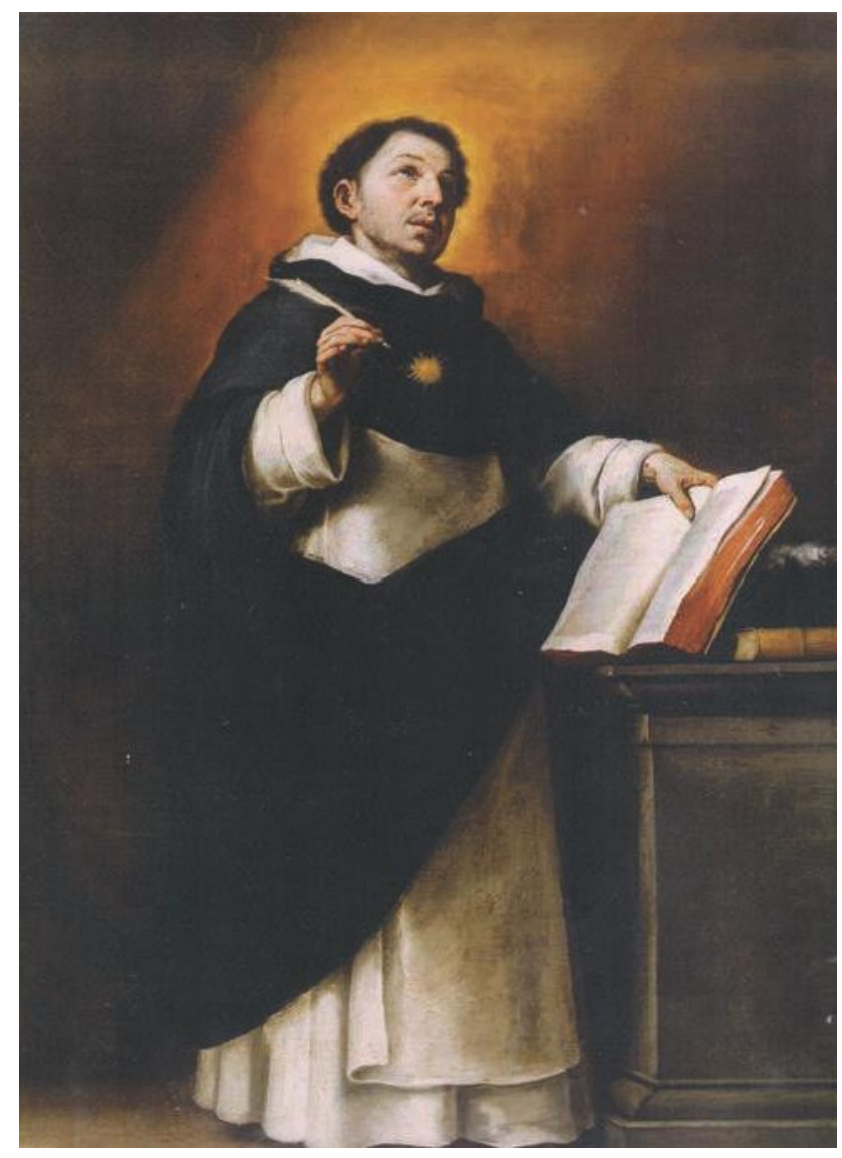

diseña ambientes de aprendizaje, que inculca la ación por el estudio y los hábitos mentales que incentivan el autoaprendizaje, el espíritu crítico, creativo y emprendedor, alguien que indica el camino, que señala alternativas y sugiere pautas de acción, sin sustituir jamás a quien aprende.

Para inclinar a los hombres a la búsqueda de la verdad y a la realización de su plenitud personal, la vía más ecaz, que no excluye sino que integra la doctrina, es el ejemplo. Se educa más por lo que se es que por lo que se dice. De este modo, el docente no solamente debe tener autoridad epistemológica, sino que debe tener también autoridad moral que la respalde o la refuerce, porque también enseña con actitudes, con la cosmovisión que tiene, con la losofía de la vida que inspira su quehacer educativo. Santo Tomás reconoce que se cree menos en las palabras que en las obras. La primera forma de enseñanza es el ejemplo, y lo más importante es la coherencia entre lo que se dice y lo que se hace. Uno se mueve a obrar incitado más por lo que ve en otro que por lo que él dice. Las palabras persuaden, los ejemplos arrastran. Todo maestro tiene que estar convencido que no hay medio más ecaz para mover al bien, que los ejemplos. Los malos ejemplos corrompen las buenas costumbres, los buenos incitan a seguirlos.

Se sabe que la palabra "maestro" viene del latín "magister", cuya raíz es la expresión "magis" que quiere decir "más", "excelente, "máximo", es decir que aquí hay una alusión a lo que se espera del portador de esa condición. ¿Quién realmente merece que se le llame maestro? No necesariamente alguien que tiene esa profesión y a quien se le paga por enseñar. Un docente no puede conformarse con enseñar, con dictar una materia. La educación no se reduce a impartir ciertas materias, como matemáticas o literatura, sino en preparar a una persona para que dé lo mejor de sí misma y viva su vida de la mejor manera posible. El maestro enseña una concepción de la disciplina o ciencia, una forma de mirarla, una losofía de la vida. Educar es cautivar con argumentos convincentes, entusiasmar por los hábitos positivos y por los valores morales, seducir con la excelencia. Esto implica comunicar conocimientos y promover actitudes. A veces queremos una educación que simplemente haga buenos profesionales y buenos operarios, pero lo que necesita la sociedad es sobre todo valientes ciudadanos y lúcidos seres humanos con opiniones propias, serias, ponderadas, que no sean sujetos de la manipulación de tantos poderes e intereses que hoy controlan el mundo.

En resumen, quienes se dedican a la enseñanza tienen que ser más maestros que docentes.

Sean maestros de sus alumnos y no sólo docentes. Dedíquenles todo el tiempo que sea necesario, sin tasarlo mezquinamente. Prolonguen la lección en el trato personal con sus alumnos, estimulen en el trato personal con 
ellos la pasión por el saber, el deseo de aspirar a metas más altas, de no conformarse con los logros adquiridos. Demuéstrenles con su vida que es posible realizar la síntesis entre el conocimiento y el amor: que a un mayor conocimiento del mundo y de la realidad, corresponde una vida moral más íntegra, que saber más signica también ser más sabio y, por tanto, mejor (Poupard, 2005).

Sólo a quienes han sido realmente maestros, se les recuerda con gratitud durante toda la vida.

\section{Referencias}

Aristóteles. Trad. Azcárate, P. (1996). Política. Madrid: Espasa Calpe.

Chesterton, G.K. (1996). Santo Tomás de Aquino. Argentina: Lumen.

Gardner, H. (2012). Verdad, Belleza y Bondad, reformuladas para la enseñanza de las virtudes en el siglo XXI. Barcelona: Paidós.

Libanio, J.B. (2003). El arte de formarse. Salamanca: Sígueme.
Martínez, E. (2002). Persona y educación en Santo Tomás. Madrid: Fundación Universitaria Española.

Martínez, E. (2004). Ser y Educar. Fundamentos de pedagogía tomista. Bogotá: Universidad Santo Tomás.

Ospina, W. (2012). La lámpara maravillosa. Cuatro ensayos sobre educación y un elogio de la lectura. Bogotá: Random House Mondadori.

Poupard, P. (2005). Santo Tomás de Aquino y la vocación de la universidad católica. Universidad Católica Argentina: Buenos Aires.

Rojas, E. (1999). La conquista de la voluntad. Bogotá: Planeta.

Rojas, E. (2004). Los lenguajes del deseo. México: Planeta.

Santo Tomás (1996). Suma contra gentiles. Madrid: BAC.

Santo Tomás (1996). Suma teológica. Madrid: BAC.

Schillebeeckx, E., OP (1965). Dieu et l'homme. Aproches Théologiques. Bruxelles: CEP.

Sedano, J.J., OP (2012). Hacia una pedagogía de la respuesta. Bucaramanga: Universidad Santo Tomás.

Cómo citar este artículo:

Corchuelo, F. Reexiones sobre la educación integral a partir del pensamiento de Santo Tomás de Aquino

Espiral, Revista de Docencia e Investigación. 2, (1), 79 - 92 
